\title{
Hybrid versus fusion imaging: are we moving forward judiciously?
}

\author{
Luca Giovanella - Giovanni Lucignani
}

Published online: 10 February 2010

(C) Springer-Verlag 2010

\begin{abstract}
"Whenever a theory appears to you as the only possible one, take this as a sign that you have understood neither the theory nor the problem which it was intended to solve" [1].
\end{abstract}

\section{Karl Popper}

(1902-1994)

Never before has medical practice been as dependent as it is today on the use of biomedical imaging technologies which, by exploiting various forms of energy, make it possible to depict the morphology and function of the human body.

The development of these technologies has been driven by the demand for better, noninvasive means of acquiring clinically relevant information. Today, in the most economically privileged countries and social classes at least, the wide availability of biomedical imaging devices is one

This Editorial Commentary refers to the article http://dx.doi.org/ 10.1007/s00259-010-1378-4

L. Giovanella

Department of Nuclear Medicine and PET/CT Centre,

Oncology Institute of Southern Switzerland,

Bellinzona, Switzerland

\section{G. Lucignani $(\triangle)$}

Department of Biomedical Sciences and Technologies and Center of Molecular and Cellular Imaging (IMAGO),

University of Milan,

Milan, Italy

e-mail: giovanni.lucignani@unimi.it

\section{G. Lucignani}

Department of Diagnostic Services, Unit of Nuclear Medicine,

San Paolo Hospital,

Via Antonio di Rudinì, 8,

20142 Milan, Italy of the main factors involved in medical decision-making processes. It is, therefore, not surprising that on two occasions the Nobel Prize for Medicine or Physiology has been awarded not to physicians, but to scientists in the hard sciences and technologies (physics, chemistry and engineering), involved in the development of imaging techniques. Thirty years ago it went to the inventors of $\mathrm{X}$-ray computed tomography (CT) and as recently as 6 years ago to the inventors of magnetic resonance imaging (MRI). Indeed, the crucial role of imaging in medicine was quickly recognised and has continued to be appreciated over the years.

The value of these inventions is encapsulated in the simple words of one of the inventors of MRI, Nobel Laureate Sir Peter Mansfield: "In many cases accurate diagnosis together with surgical and/or drug intervention indicates a brighter future beyond the illness. Of the many letters received recently what comes through in much of the correspondence is the strong sense of relief at knowing the details of their illness and the hope inspired by the rigorous evaluation of their problem using MRI. Many patients offer their profuse thanks that such machines now exist" [2].

Thanks to the concurrent efforts of scientists from a broad spectrum of disciplines, the development of X-ray $\mathrm{CT}$ and MRI has been paralleled by the evolution of tomographic radionuclide imaging techniques, although the advances in this latter field have yet to receive the acknowledgement they deserve.

The use and integration of the different imaging technologies should be guided primarily by the principles of justification and optimization, particularly when the use of ionizing radiation is involved. But cost-effectiveness, too, is an important consideration, calling for adequate management strategies. These strategies vary widely and are based on a number of variables: the mission as well as 
the setup of the individual clinical centre, the type of patients being examined, workflows and patient throughput, to mention just a few. The progressive integration in the clinical setting of the various evolving imaging modalities was a natural and predictable development given that these technologies, in spite of their many differences, share a common purpose: the representation in images of organs and processes in the body.

Different approaches to the integrated use of imaging techniques have been adopted, but basically they come down to two strategies. Initially, integration was based on the fusion, using dedicated software, of images obtained separately; subsequently, hybrid machines were introduced that allow the almost simultaneous acquisition, i.e. in rapid sequence, of diagnostic images. Each of these two approaches seems to have different advantages and drawbacks, and both demand considerable levels of investment in order to meet the specific needs of different centres and applications.

\section{Image integration software and hardware: the state of the art}

The combining of images derived from different modalities into a single, enhanced image with inherent added clinical value is already a well-developed approach; consequently, the pace of its evolution has now slowed down. By contrast, the "hybridization" of different imaging machines, far more complex and costly, is an approach still under rapid and continuous evolution. Whereas computing power allows affordable, flexible, real-time image fusion using technologies that are independent of the imaging systems themselves, the combination of different pieces of equipment still presents significant technological and economic problems. Indeed, while the difficulties of integrating X-ray $\mathrm{CT}$ and PET, as well as X-ray CT and SPECT machines have been overcome brilliantly, the integration of PET and MRI, in spite of the considerable advances achieved thus far, still has a long way to go. Furthermore, the integration of two pieces of hardware is based on technology that ages quite rapidly as new models of the individual imaging instruments are independently developed. The upshot is that the technology of hybrid machines lags behind that of the individual imaging techniques, and its development is a major undertaking in terms of time and money. Thus, to appreciate the relative merits, with respect to efficiency and effectiveness, of hybrid imaging versus image fusion, a number of aspects have to be taken into account.

It goes without saying that what we want (and the patient too!) is the most reliable clinical assessment possible, performed by the best clinical teams using the best available clinical tools or combination of tools. Crucial to the achievement of this objective is the ability to ask the right questions in order to identify the right biological target and, consequently, the most appropriate tools. Appropriateness is a key issue, particularly when the use of radiation sources is involved, and repeated examinations involving irradiation need to be avoided. In addition, of course, the combination must be economically sustainable, efficient and effective. In the light of these considerations, various questions arise: do we need hybrid imaging acquisition all the time? Can we manage with just fusion? How can we best exploit both options?

\section{Hybrid machinery}

At this point, it is worth recalling why hybrid instruments such as PET/CT were developed in the first place. Adding a CT system to a PET device offers two advantages: first, the possibility of obtaining attenuation correction of PET data using CT attenuation coefficients, and second, the possibility of visualizing all the PET-derived biochemical information in a single morphological volume. From this latter perspective, since the use of CT is ancillary to PET and not necessary to it for diagnostic purposes, the acquisition of diagnostic-quality $\mathrm{CT}$ images is not a requirement and an unenhanced low-dose CT examination can be performed. In fact, both of the above objectives can be achieved using just one CT detector ring, although the advantages of multiple rings (at least 32) emerge strongly in particular cases, for example when there is a need for gating acquisition in respiratory and cardiac studies. It is important to be aware that software image registration is also necessary when using hybrid PET/CT imaging systems in which the acquisition is not simultaneous but sequential, albeit performed without moving the patient from the bed.

\section{Multimodality image fusion}

As remarked by Slomka and Baum, the software registration of stand-alone scans "paved the way" for the clinical application of hybrid scanners, demonstrating the practical benefits of image integration before the hybrid dualmodality devices became available [3]. Nowadays software registration techniques play a significant role in patientspecific, cost-effective, and radiation dose-effective applications of integrated imaging. Software techniques allow accurate $(2-3 \mathrm{~mm})$ rigid image registration of brain PET with CT and MRI. Nonlinear techniques, required for multimodality radiation planning, are used in whole-body image registration and recent developments in this field now allow significantly accelerated computing times. As these authors remark, difficulties remain in the validation of 
nonlinear registration of soft-tissue organs. The use of software-based multimodality image integration in a clinical environment is sometimes hindered by a lack of the picture archiving and communication systems (PACS) infrastructure needed to integrate efficiently and automatically all available images into one common database.

\section{Contrast-enhanced studies with PET/CT hybrid cameras}

While contrast-enhanced PET/CT (PET/ceCT) is hailed by some authors as the ideal "one-stop shop" imaging procedure in oncology, its impact on patient management and cost effectiveness are still largely unproven. First, a tumour's response to therapy is assessed mainly on the basis of functional and clinical data rather than morphology. Consequently, low-dose, nonenhanced CT could be sufficient in oncological patients, who account for a large proportion of the population referred for PET/CT examinations [4]. Second, PET/ceCT has shown no added value when compared with nonenhanced PET/CT in the pretreatment evaluation (i.e. staging) of diffuse tumours such as lymphomas and head and neck cancers.

Schaefer et al. retrospectively compared the diagnostic value of coregistered PET/CT scans using both low-dose nonenhanced $\mathrm{CT}$ and contrast-enhanced $\mathrm{CT}$ (ceCT) protocols in the disease staging and restaging of 60 patients with Hodgkin's disease or high-grade non-Hodgkin's lymphoma [5]. These authors concluded that ceCT can, if necessary, be performed in any desired location and used for image fusion. However, PET/CT performed with low-dose CT and without intravenous contrast medium is sufficient for disease staging and restaging of patients with Hodgkin's disease and high-grade non-Hodgkin's lymphoma.

Yoshida et al. evaluated whether the use of intravenous contrast medium in integrated ${ }^{18} \mathrm{~F}-\mathrm{FDG}-\mathrm{PET} / \mathrm{CT}$ significantly contributes to the evaluation of primary head and neck cancers compared with nonenhanced PET/CT, regional ceCT of the head and neck, and regional MRI of the head and neck. No statistically significant difference was found between PET/CT and neck MRI in T-status classification [6]. Globally, an almost perfect correlation between enhanced and nonenhanced PET/CT was found for lesion detection and initial staging of primary head and neck cancers, leading the authors to conclude that routine contrast agent administration in PET/CT imaging may not be justified.

Conversely, contrast enhancement may be of value in the PET/CT assessment of liver metastases, especially before resective surgery. Soyka et al. compared PET/ceCT, ceCT alone and nonenhanced PET/CT in 54 patients with recurrent colorectal cancer. Compared with nonenhanced
PET/CT, PET/ceCT provided additional information in 39 of the 54 patients $(72 \%)$; this information had therapeutic relevance in 23 patients. This large number was primarily due to correct segmental localization of liver metastases, which is crucial for surgical therapy planning [7].

These data are further confirmed by Kitajima and colleagues in a series of studies in patients with uterine, ovarian, colorectal and pancreas cancer [8-13].

Taken together, all these findings indicate that ceCT (either performed separately or built into a PET/CT protocol) may be of value in selected cases, especially when surgical planning is needed and a fine definition of vascularity is required. In these instances, the decision as to whether to perform ceCT and PET/CT separately or instead to proceed directly with a PET/ceCT protocol is largely dependent on local resources and organization. It is important to note that patients who have undergone ceCT shortly before being referred for PET/CT may not need additional ceCT during the PET/CT study if the previous dataset is available for image correlation.

\section{The clinical question and the biological targets behind the simultaneous use of two modalities}

The above example shows that the way forward lies in the continued evolution of fused and hybrid anatomical and molecular imaging techniques, using both existing and new radiopharmaceuticals that image specific targets in disease processes. Whether the information is obtained by fusion or by the hybrid approach is a secondary issue; what really matters is the biology of the process. In other words, the emphasis needs to be on the information being sought, not on the technology used to seek it. After all, with new biomarkers being developed all the time, strategies and instruments that are valid today may not be adequate a few years from now. For example, whereas static acquisitions are the rule with the tracers presently available in oncology, cardiology and neurology, some radiopharmaceuticals could turn out to need dynamic acquisitions, and this would mean the X-ray CT equipment in the hybrid machine remaining on standby for hours.

In a recent paper, von Schulthess and Schlemmer set out to distinguish the facts from the fiction concerning these integrated systems [14], reviewing current experience of combined imaging using existing brain PET/MRI and PET/CT systems. They discussed various types of PET and MRI integration: both completely independent systems and completely integrated systems offering the possibility of simultaneous data acquisition. They also looked at what simultaneous data acquisition with nuclear imaging systems combined with MRI or CT really means, 
given that the pharmacokinetics of the nuclear tracers used may make technical simultaneity irrelevant. These authors conclude that combining PET/MRI is an interesting research endeavour, but one whose outcome is uncertain. They argue that while completely simultaneous brain applications are of research interest immediately, clinical applications do not currently warrant the construction of fully integrated systems. The use of adjacent systems where imaging tables are linked with a patient "shuttle", thereby requiring only patient translation, not repositioning, may be a good point from which to start assessing the value of integrated PET/MRI.

As Antoch and Bockisch point out, both PET and MRI examination times are substantially longer than those of CT alone [15]. Efforts to combine PET and MRI in a single imaging modality must produce solutions that offer competitive examination times. According to these authors, such solutions will probably have to be based on simultaneous acquisition of MRI and PET, otherwise two already long procedures would add up to an unacceptably protracted examination time, incompatible with the clinical setting. To be clinically competitive in terms of costeffectiveness and acceptability to patients, the examination time of a PET/MRI protocol should not substantially exceed that of a PET/CT one. Another clinically important and often overlooked issue relating to lengthy PET/MRI acquisitions is the risk of patient motion leading to inaccuracies in the fusion of PET/MRI data.

\section{The limitations of hybrid PET/CT systems as diagnostic tools and the need for PET and MRI data}

Whereas PET/CT will continue to have a role in attenuation correction and localization, the combination of these two modalities could prove inadequate for clinical purposes in some cases. Kim et al., for example, evaluated the additional diagnostic value of MRI/PET fusion compared with PET/CT in the detection of metastatic lymph nodes in cervical cancer patients [16]. This is a case in which the fusion of two modalities provides more accurate information for cancer management.

Within a scientific setting, in which it naturally falls to us to look beyond achieved goals, and in particular as the use of PET/CT progressively becomes routine, we should be looking for new research targets besides, for example, new tracers. We should be aiming to develop Star Trekstyle "sick-bay scanners", in which patients are submitted to a single diagnostic examination-a PET/CT/MRI scan. Such a development would be welcomed by patients, sparing them the need to return for further imaging procedures. Obviously, this will come with a price tag, but it is a price that could be sustainable.

\section{Are there any limits to image fusion and hybrid machines?}

Although the most popular image integration applications are based on PET and CT, other applications involve substantially all modalities. A recent example was provided by Walimbe et al., who implemented a new multimodality stress test for accurate correlation of complementary functional and perfusion data from real-time threedimensional (3-D) echocardiography and SPECT, respectively [17]. Compared with the angiographic findings in 12 patients, the diagnostic approach based on fused images correctly identified 13 of 13 diseased arteries, compared with 11 of 13 identified by SPECT and 6 of 13 identified by echocardiography-based analysis. The authors conclude that their results support the feasibility and potential effectiveness of novel multimodality stress testing. Clearly, this type of integration would not be feasible using a hybrid approach.

\section{Coregistration of images in radiotherapy planning}

The coregistration of images acquired using PET and CT scanners is also gaining importance in the field of radiotherapy (RT) planning. Grgic et al. recently pointed out that the RT planning process is often based on separately acquired FDG-PET/CT images and planning CT scans [18]. In clinical practice, patients referred to the radiotherapist often present with previously acquired FDGPET or PET/CT scans. However, the patient positions and breathing protocols used for diagnostic scans often differ from those used in CT simulation and RT treatment. These authors list several ways of incorporating PET images into the RT planning process: (1) PET and CT images from separate scanners may be coregistered using software; (2) PET/CT images from a combined scanner may be coregistered with planning CT scan images; or (3) a separate planning PET/CT scan can be obtained. In cases 1 and 2, it may, for organizational and economic reasons, be desirable to use FDG-PET image data acquired using a diagnostic protocol undertaken before RT planning. However, anatomical misalignment, caused mainly by respiration and differences in patient positioning, can significantly jeopardize the image interpretation, resulting in false localization of FDG-positive lesions and therefore of RT target volumes. Grgic et al. compared intraindividual differences between PET scans acquired in diagnostic (D-PET) and RT treatment (RT-PET) positions coregistered with planning $\mathrm{CT}$ images acquired using different breathing protocols in 16 patients with non-small-cell lung carcinoma. These authors concluded that significantly better fusion of PET and planning CT images can be achieved with PET images 
acquired in the RT position. The best intraindividual fusion results were obtained with the planning $\mathrm{CT}$ scan performed during mid-breath hold. Globally, a separate planning PET scan is justified when only a diagnostic PET or PET/CT scan is available. Ideally, providing there is close interdepartmental cooperation, diagnostic FDG-PET scans of RT candidates might routinely be performed using positioning aids and mid-breath hold, precisely in order to avoid the need for a second PET scan.

\section{Costs and workflow analysis of fusion in comparison with hybrid systems}

While it is not unusual to scan four to five patients per hour using the commonly available CT cameras, a CT camera linked to a PET scanner would instead produce only 12 , at most 15 , scans a day for subsequent attenuation correction and localization. What is more, using the $\mathrm{CT}$ component needed to perform contrast-enhanced studies would further reduce the number of PET scans obtainable each day and also lead to suboptimal use of the (rapidly decaying) available radiopharmaceutical.

Thus, whereas a four- to eight-slice CT scanner would be just about sufficient for the purposes of attenuation correction and localization of PET findings, and for carrying out occasional diagnostic studies with or without contrast enhancement, thereby justifying the costs incurred, the immobilization of scanners offering 64 slices or more for the entire duration of PET scanning, and vice versa, would generate high costs per patient examined. This also applies to the combination of PET and MRI.

Von Schulthess and Burger recently looked at the fusion versus hybrid systems approach (based, respectively, on the use of sequential in comparison with simultaneous data acquisition) from the cost and workflow perspective, analysing these costs as a function of overall system costs and imaging times [19]. From the workflow perspective, an advantage of integrated systems is that patients have to be up- and downloaded only once. However, if the extra imaging time required by an integrated system exceeds the time it takes to switch them between devices in a separate image acquisition protocol, then this advantage is lost, making separate data acquisition preferable. On the cost side, integrated systems emerge as advantageous when their overall cost amounts to only three-quarters, or less, of that incurred by the use of separate systems. On this basis, the authors reached the conclusion that PET-CT scanning is a cost-effective implementation of an integrated system, whereas most current SPECT-CT systems are not costeffective. Integration of two devices in two rooms by means of a shuttle may be a way of making PET-MR cost-effective and may also be a design option for SPECT-CT systems.

\section{Conclusion}

It is customary in clinical practice for physicians to integrate data from various disciplines obtained using a range of different technologies. Indeed, a diagnosis can be based on hundreds of laboratory findings and pathological specimen assays, reviewed in the light of clinical findings. The same applies within the field of imaging. As noted by the inventor of PET/CT, David Townsend, the extensive development of image fusion techniques over the past 20 years has shown that the fusion of images from complementary modalities offers a more complete and accurate assessment of disease than the fusion of images from a single modality [20]. Although software fusion has its limitations (software techniques have been successful in fusing images of the brain acquired using different modalities, but less so in fusing images of other parts of the body) many of these have been addressed by the recent introduction of technology that can acquire both anatomical and functional images in a single scan. The combination of CT and PET was introduced commercially in 2001. Clinical adoption of PET/CT has been surprisingly rapid, and despite continuing debate, the new technology has advanced the use of clinical molecular imaging, particularly in oncology.

What emerges clearly from Townsend's words is that "hybrid" is the essence of image fusion, and also the key to further developments in this field. However, this is not to say that we should be using exclusively hybrid machines to perform the whole and wide range of investigations that a top non-hybrid machine could perform (and indeed Townsend himself has never suggested this). The use of hybrid machines may not always be cost-effective; in fact, if lowdose CT within a hybrid machine is sufficient for lesion localization, this $\mathrm{CT}$ image could successfully be fused with diagnostic CT scans or MRI, or echographic and angiographic studies.

One of the blessings of today's "new" technology is that it has not only strengthened the position of nuclear medicine (now molecular imaging) and boosted net profit, but also opened up this specialty to the interest of radiologists, who might claim that this technology is not fully exploited in the hands of nuclear medicine physicians. Clearly, when a hybrid scanner is available it is very tempting to exploit it to the full and in many circumstances its use is highly appropriate for a thorough patient work-up and not only for attenuation correction. Although this dilemma has not yet been critically appraised through systematic comparisons of the fused and hybrid approaches, and more could be done in this regard, existing data nevertheless suggest that fused imaging is not as good as hybrid imaging, and that contrastenhanced imaging is better than non-contrast-enhanced imaging in selected cases - arguments that are, of course, 
widely used for marketing purposes. However, although cost-effectiveness studies are not yet widely available, it must be remembered that neither hybrid nor fused imaging can be carried out at no cost.

There remain two other issues that warrant consideration: dosimetry and competence. As regards the first of these, it is clear that, when possible, fusion of available studies is recommended in order to avoid multiple exposures to ionizing radiation. To this same end, a low-dose CT scan along with a PET study could be a reasonable compromise for obtaining images suitable for diagnostic image fusion. As far as the issue of competence is concerned, the continued advance of biomedical imaging technologies demands continuous expansion of our knowledge so that we are equipped to exploit to the full the potential offered by the various techniques and methods now available for studying different organs with different diseases. From this perspective, it can be argued that the application of PET and CT within a single hybrid system, or in a "fused mode" involving two independent, separate systems, demands in-depth knowledge of both these techniques and of their clinical use. There is a growing number of specialists trained both in radiology and nuclear medicine who claim that the very availability of hybrid systems makes their use in the full range of options mandatory.

We have thus reached a crossroads, at which expertise and self-criticism must be employed as we seek to identify the direction we should be moving in, both as a specialty and in our capacity as individual nuclear physicians. It is a hard-fought issue. It can be remarked that whereas nuclear medicine training, practice and excellence have been somehow circumscribed to a handful of highly frequent conventional nuclear medicine imaging procedures, similar excellence in the field of PET/CT imaging has not been reached yet by many nuclear medicine specialists and training programmes. Thus, today, nuclear physicians are required to know far more about oncology than they did before hybrid imaging came along as a high-throughput diagnostic tool. Nuclear medicine physicians also need to learn to appreciate the broad spectrum of CT findings and differential diagnoses, e.g. "tracer-cold" lesions (which radiologists frequently cite in their reports since they often show high sensitivity but lower specificity), which is not the case for the most conventional "hot-spot"-driven nuclear medicine approach. It must be admitted that due to their background, it might be easier for radiologists specialized in oncological imaging to learn the PET part than for nuclear medicine physicians to learn radiology exnovo and in depth.

Despite the existence of numerous regulatory barriers it is at this point possible to envisage the creation of a new subspecialty for the proper interpretation of hybrid and fused imaging by a single specialist, and not by two separate individuals. This is one of the most critical issues in the field of hybrid/fused/integrated imaging, quite apart from whether it is really needed or not.

However, although it is easy, in this setting, to wonder whether it is now becoming necessary to "hybridize" and "fuse" the brains of new generations of radiologists and nuclear medicine physicians, it is also necessary to appreciate that this could lead to an unacceptable reduction in their specificity, sensitivity and overall ability to keep up with technological evolutions. It is one thing to say "I can do it", but quite another to be able to say "I can do it well and efficiently", as the patient expects. To this end, the pursuit of the full hybrid approach would probably require complete rethinking of training and residency programmes, to render them more geared towards forming experts in the overall integrated diagnostic process, more deeply "organ" or "pathology" oriented, and less technology oriented, than is the case now.

Nobel Prize winner Allan Cormack made a candid remark in his Nobel lecture: "There is irony in this award, since neither Hounsfield nor I is a physician. In fact it is not much of an exaggeration to say that what Hounsfield and I know about medicine and physiology could be written on a small prescription form!" [21] — this is, of course, very probably an exaggeration. Nevertheless, Cormack's statement invites us to reflect upon the fact that when he and Hounsfield invented CT, and Mansfield and Lauterbourg invented MRI, they all had only a small idea of these machines' enormous clinical potential. In short, it is up to us, as clinicians, to remember that, whatever remarkable combination of machines an enthusiastic vendor may present us with, we have a duty to respond to precise clinical needs, in a precise clinical context, regardless of the beauty of the toys, and to optimize judiciously the use of these instruments. In particular we should carefully avoid the repetition within a short time of a ceCT, which may be a temptation just because the patient is already there, in the PET/CT machine, for a PET study following a previously performed ceCT on a separate machine.

Acknowledgments The authors are grateful to PD Dr. Thomas Hany of the Klinik und Poliklinik für Nuklearmedizin, Universitätsspital Zürich, for fruitful discussion and to Ms. Catherine Wrenn for her advice and editorial support.

\section{References}

1. Popper KR. Objective knowledge: an evolutionary approach. USA: Oxford University Press; 1972.

2. Nobelprize.org. Sir Peter Mansfield: Banquet Speech. http:// nobelprize.org/nobel_prizes/medicine/laureates/2003/mansfieldspeech.html

3. Slomka PJ, Baum RP. Multimodality image registration with software: state-of-the-art. Eur J Nucl Med Mol Imaging 2009;36 Suppl 1:S44-55.

4. Kuehl H, Veit P, Rosenbaum SJ, Bockisch A, Antoch G. Can $\mathrm{PET} / \mathrm{CT}$ replace separate diagnostic CT for cancer imaging? 
Optimizing CT protocols for imaging cancers of the chest and abdomen. J Nucl Med 2007;48 Suppl 1:45S-57S.

5. Schaefer NG, Hany TF, Taverna C, Seifert B, Stumpe KD, von Schulthess GK, et al. Non-Hodgkin lymphoma and Hodgkin disease: coregistered FDG PET and CT at staging and restaging-do we need contrast-enhanced CT? Radiology 2004;232(3):823-9.

6. Yoshida K, Suzuki A, Nagashima T, Lee J, Horiuchi C, Tsukuda $\mathrm{M}$, et al. Staging primary head and neck cancers with (18)F-FDG $\mathrm{PET} / \mathrm{CT}$ : is intravenous contrast administration really necessary? Eur J Nucl Med Mol Imaging 2009;36(9):1417-24.

7. Soyka JD, Veit-Haibach P, Strobel K, Breitenstein S, Tschopp A, Mende KA, et al. Staging pathways in recurrent colorectal carcinoma: is contrast-enhanced 18F-FDG PET/CT the diagnostic tool of choice? J Nucl Med 2008;49(3):354-61.

8. Kitajima K, Murakami K, Yamasaki E, Domeki Y, Tsubaki M, Sunagawa M, et al. Performance of integrated FDG PET/contrastenhanced $\mathrm{CT}$ in the diagnosis of recurrent colorectal cancer: comparison with integrated FDG PET/non-contrast-enhanced CT and enhanced CT. Eur J Nucl Med Mol Imaging 2009;36(9):1388-96.

9. Kitajima K, Murakami K, Yamasaki E, Kaji Y, Shimoda M, Kubota $\mathrm{K}$, et al. Performance of integrated FDG-PET/contrast-enhanced CT in the diagnosis of recurrent pancreatic cancer: comparison with integrated FDG-PET/non-contrast-enhanced CT and enhanced CT. Mol Imaging Biol 2009. doi:10.1007/s11307-009-0271-7

10. Kitajima K, Murakami K, Yamasaki E, Kaji Y, Sugimura K. Accuracy of integrated FDG-PET/contrast-enhanced CT in detecting pelvic and paraaortic lymph node metastasis in patients with uterine cancer. Eur Radiol 2009;19(6):1529-36.

11. Kitajima K, Murakami K, Yamasaki E, Domeki Y, Kaji Y, Morita $\mathrm{S}$, et al. Performance of integrated FDG-PET/contrast-enhanced $\mathrm{CT}$ in the diagnosis of recurrent uterine cancer: comparison with PET and enhanced CT. Eur J Nucl Med Mol Imaging 2009;36 (3):362-72.

12. Kitajima K, Murakami K, Yamasaki E, Kaji Y, Fukasawa I, Inaba $\mathrm{N}$, et al. Diagnostic accuracy of integrated FDG-PET/contrast- enhanced CT in staging ovarian cancer: comparison with enhanced CT. Eur J Nucl Med Mol Imaging 2008;35(10):1912-20.

13. Kitajima K, Murakami K, Yamasaki E, Domeki Y, Kaji Y, Fukasawa I, et al. Performance of integrated FDG-PET/contrastenhanced $\mathrm{CT}$ in the diagnosis of recurrent ovarian cancer: comparison with integrated FDG-PET/non-contrast-enhanced CT and enhanced CT. Eur J Nucl Med Mol Imaging 2008;35 (8):1439-48.

14. von Schulthess GK, Schlemmer HP. A look ahead: PET/MR versus PET/CT. Eur J Nucl Med Mol Imaging 2009;36 Suppl 1: S3-9.

15. Antoch G, Bockisch A. Combined PET/MRI: a new dimension in whole-body oncology imaging? Eur J Nucl Med Mol Imaging. 2009;36 Suppl 1:S113-20.

16. Kim SK, Choi HJ, Park SY, Lee HY, Seo SS, Yoo CW, et al. Additional value of MR/PET fusion compared with PET/CT in the detection of lymph node metastases in cervical cancer patients. Eur J Cancer 2009;45:2103-9

17. Walimbe V, Jaber WA, Garcia MJ, Shekhar R. Multimodality cardiac stress testing: combining real-time 3-dimensional echocardiography and myocardial perfusion SPECT. J Nucl Med 2009;50(2):226-30.

18. Grgic A, Nestle U, Schaefer-Schuler A, Kremp S, Kirsch CM, Hellwig D. FDG-PET-based radiotherapy planning in lung cancer: optimum breathing protocol and patient positioning - an intraindividual comparison. Int J Radiat Oncol Biol Phys 2009;73 (1):103-11.

19. von Schulthess GK, Burger C. Integrating imaging modalities: what makes sense from a workflow perspective? Eur J Nucl Med Mol Imaging doi:10.1007/s00259010-1378-4.

20. Townsend DW. Dual-modality imaging: combining anatomy and function. J Nucl Med 2008;49(6):938-55.

21. Nobelprize.org. Allan M. Cormack: Banquet Speech. http:// nobelprize.org/nobel_prizes/medicine/laureates/1979/cormackspeech.html 